\title{
Substitution in Smartphone Communication Services
}

\author{
Juuso Karikoski \\ Department of Communications and Networking \\ Aalto University \\ Espoo, Finland \\ juuso.karikoski@aalto.fi
}

\author{
Sakari Luukkainen \\ Department of Computer Science and Engineering \\ Aalto University \\ Espoo, Finland \\ sakari.luukkainen@aalto.fi
}

\begin{abstract}
Substitution between mobile internet communication services and traditional mobile operator-provided communication services is studied in this paper using smartphones. The empirical data are collected with handsetbased measurements conducted in Finland between 2008 and 2010. The data are collected from a total of 183 early adopter Symbian smartphone users. The individual-level correlation analysis indicates that no clear evidence in favor of substitution can be found. This implies that the services are used for different purposes as independent services and are not direct substitutes to each other. The results support similar studies conducted by other researchers. However, the analysis can be extended and improved in a number of ways that are discussed as future research.
\end{abstract}

Keywords - substitution; smartphones; communication services; handset-based measurements

\section{INTRODUCTION}

The usage and traffic generated by Mobile Internet (MI) services have exploded in the recent years. This, together with the flat-rate pricing schemes, has generated problems for mobile operators, who have been forced to make network investments without increasing their revenue substantially. Internet services like Gmail, Skype and Facebook have started to disrupt mobile operators' business models, which are traditionally based on a walled garden principle. The voice and SMS (Short Message Service) services still generate most of the operators' revenues today, but these traditional services have been commoditized and ARPU (Average Revenue per User) figures have decreased significantly because of the fierce competition. The operators suspect that the new MI communication services might substitute their voice and SMS / MMS (Multimedia Messaging Service) services and this has affected their marketing strategy decisions. Thus the operators need to decide whether to adopt the emerging, possibly substituting, services and be exposed to the risk of cannibalization, or whether not to integrate and be exposed to the risk of increasing customer churn. That is why it is crucial for the operators to understand the cross elasticities between the services.

The aim of this paper is to study handset-based data gathered between 2008 and 2010 in Finland to explore if there is empirical evidence in favor of substitution in smartphone communication services. We consider smartphone Operating Systems (OS) as the platforms and Symbian as the platform under study. The key feature for smartphones in this study is the capability to install third party application software. At the moment of data collection Symbian was the dominating platform in terms of market share according to Gartner [1]. However, Symbian has been losing its market share rapidly while other platforms such as Google Android and iPhone are gaining market share. The services analyzed in this study are divided to traditional communication services and MI communication services. The traditional services include cellular voice calls, SMSs and MMSs. The MI services on the other hand contain all internet-based operator-independent mobile communication services, such as email, Instant Messaging (IM), Voice over IP (VoIP) and social media services.

According to the Finnish Communications Regulatory Authority (Ficora) [2] the absolute numbers of calls, SMSs and MMSs have increased steadily in the Finnish mobile communications market since 2008. However, the number of mobile subscriptions has increased even more in proportion, resulting in a decreasing number of calls, SMSs and MMSs per subscription. The subscriptions include also mobile broadband subscriptions, which have more than tripled during the las three years in Finland. These subscriptions are used variably for calling and messaging purposes, however, and thus more detailed statistics are needed for drawing any conclusions on possible substitution on market-level. Furthermore, as Gerpott [3] has indicated, these kinds of aggregate level analyses may mask diverging individual level change patterns in different customer segments. For example, they do not separate the effect of customers who have not yet even tried MI services. Thus some micro level analysis is required among users who have experience in using MI communication services.

There has been a very limited amount of research conducted in this area of substitution which is based on empirical behavioral data on individual level. Verkasalo [4] gathered handset-based data from early adopter smartphone users during 2005 and 2006 in Finland and analyzed the usage data in terms of session initiations. The results indicate that in 2005 and 2006 the share of MI communication from all communication was very small, only 2.04 per cent and 1.07 per cent, respectively. Also in another study utilizing the same data it was observed that only $21 \%$ of the users had used email and $5 \%$ had used IM [5]. Verkasalo's results imply that there is no clear relation between the number of SMSs generated or the frequency and length of voice calls made and the use intensity of MI multimedia services. However, Verkasalo considered MI multimedia services, whereas we consider MI communication services. It seems that it was somewhat early to study substitution in 2005 and 2006 as the users were not eager to 
adopt new MI services just yet. More recently Gerpott [3] has studied the impacts of MI use intensity on the demand of SMS and mobile voice telephony. His study did not either focus specifically on communication services; instead all MI traffic was analyzed. Our study is more detailed since the actual application usage is analyzed instead of the traffic. Gerpott [3] combined usage data from a mobile operator's billing system and telephone surveys conducted in 2008 in Germany. He concluded that the MI use intensity significantly negatively affected both the number of SMSs sent and received. The effects were so small, however, that their practical relevance is highly doubtful. Furthermore, a similar effect could not be found for outgoing or incoming voice calls. Also in another study by Gerpott [6] no significant correlation between MI use intensity and mobile voice communication or SMS activity levels was observed.

\section{THEORETICAL BACKGROUND}

Substitution can be studied from different theoretical perspectives. In economic theory complements and substitutes are usually defined in terms of cross elasticity measures. For example, cross elasticity of demand $(\varepsilon)$ between services $j$ and $k$ is defined in [7] as the percentage change in the demand $(x)$ for service $j$ per percentage change in the price $(p)$ of service $k$. Consequently:

$$
\epsilon_{j k}=\frac{\delta x_{j}(p) / \delta p_{k}}{x_{j} / p_{k}}
$$

and

$$
\frac{\Delta x_{j}}{x_{j}}=\varepsilon_{j k} \frac{\Delta p_{k}}{p_{k}}
$$

Thus, if $\delta \mathrm{x}_{\mathrm{j}}(\mathrm{p}) / \delta \mathrm{p}_{\mathrm{k}}>0$ in (1) services $j$ and $k$ are substitutes and if $\delta \mathrm{x}_{\mathrm{j}}(\mathrm{p}) / \delta \mathrm{p}_{\mathrm{k}}<0$ they are complements. If $\delta \mathrm{x}_{\mathrm{j}}(\mathrm{p}) / \delta \mathrm{p}_{\mathrm{k}}=0$ the services are independent.

Price is, however, not the only variable that the consumers base their decisions on. There might be switching costs [8], such as the cost of installing new software that prevent the switch to a cheaper or even better quality service. Also network externalities [7] might be present further increasing the switching costs. For example, there might be a smaller number of users reachable through the new service, which decreases the utility of that service to the user. Porter [8] has also coined the term buyer's propensity to switch which is a third variable influencing the consumers' decisions. Not all consumers have equal propensities to switch to a new service when faced with a comparable economic motivation.

Substitution is addressed in the marketing literature traditionally from a product perspective, but since a product is anything that can be offered to a market to satisfy a want or need [9, p. 505] we can use the product literature when considering our case of smartphone communication services. Marketing scholars talk about multiproduct interactions [10] or interproduct (or intercategory) relationships [11], for example, where substitution is one type of interaction or relationship. These interactions or relationships are presented in a conceptual framework [10] which is illustrated in Fig. 1. This framework serves as a good basis for analyzing smartphone communications services.

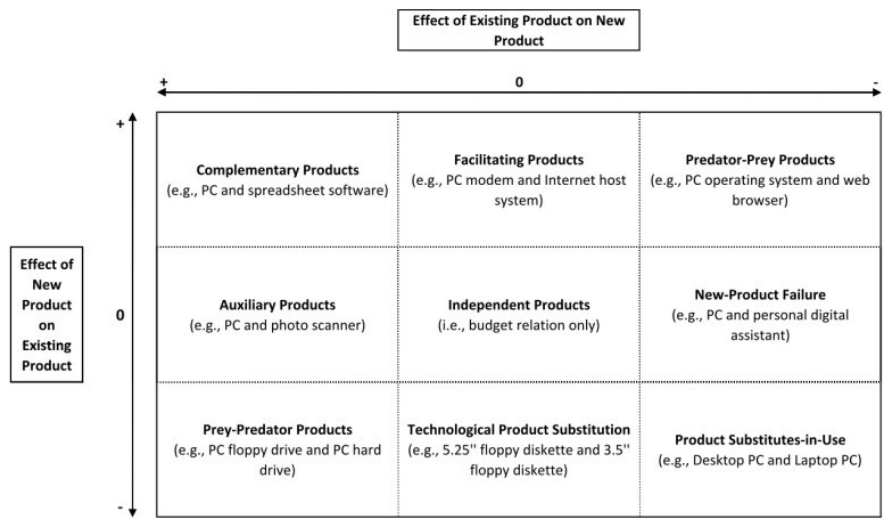

Figure 1. Conceptual framework for multiproduct interactions [10]

As the framework suggests there are multiple possible interactions between products. First of all Technological Product Substitution happens when a new and improved product replaces an older product. New-Product failure, as the name implies, is a type of interaction between an older product and a new product that results in the existing product beating the new one. In Product Substitutes-in-Use the interacting products have a negative effect on each other. Complementary Products on the other hand can enhance the growth prospects of each other simultaneously. In Facilitating Products the newer product has a positive effect on the older one, but not vice versa, whereas in Auxiliary Products the older product has a positive effect on the new one. In a Predator-Prey Situation a new product has a positive effect on an older product while the older product in turn has a negative effect on the newer product. In a Prey-Predator Situation the effects are vice versa. Lastly, Independent Products do not directly affect each other and the products are only related through their costs and the finite monetary resources of the consumers. The framework has two steady-state conditions - the products end up coexisting or only one product survives. Technological product substitution and new-product failure results in only one product surviving, while independent, complementary, facilitating and auxiliary products cases result in coexisting products. Rest of the cases can eventually lead to either of the two conditions. There is an extension to the framework in [11] which takes into account the dynamics between complements and substitutes. There might be a gradual shift from complements to substitutes in the course of time and vice versa. These modes are called reincarnation and rejuvenation, respectively. For purposes that are nonspecific the substitutes and complements might also be of an occasional nature.

The strategic management literature approaches substitution from competitive opportunities and threats perspectives, for example, how a company can defend itself against substitutes. Substitution is one of the five competitive forces of Porter [8] which determine the profitability of an industry. Porter discusses the substitution at the industry level but the same principles apply to product and service level. Industries also evolve through a sequential development of technology cycles. These cycles are initiated by the technological discontinuities, which start to substitute the existing products and related services. The technology cycle phases are technological variation, selection and retention as discussed in [12]. The threat of substitute products or services 
depends on a number of factors: the relative price, new features and added value, performance and switching costs [8]. According to Rogers [13] the most important factor affecting diffusion is the relative advantage (price and performance) over competing technology substitutes, which changes over time. Another factor affecting the diffusion is the compatibility with the values, norms, and experience of the end-users. Innovations that are connected to older, already proven technology have an advantage.

As discussed in the introduction telecommunications infrastructure requires that operators make large long-term proactive investments in a situation where the characteristics of the new market are not clear. For this reason, more knowledge is needed of substitution during an intensive technological change. New technologies significantly influence the business of incumbent operators and can easily cannibalize older services. Technological change can lead to decreasing sales of the existing services, and an unfavorable cannibalization leads to lower profits or to the total loss of that market. The risks of cannibalization involve services being less profitable and markets for them being smaller than the old one, or they might contain significant technical risks and require continuous retooling [14].

\section{METHODOLOGY AND EMPIRICAL DATA}

First of all we need to clarify how the usage of the services is measured. From all of the traditional services we can measure the actual communication, i.e., calls made and messages sent as well as the actual duration of voice calls in seconds. With MI services, however, we can only measure if and for how long the service was used. Thus we cannot measure, e.g., if messages were actually sent during the usage of the MI service or if the service was just used for reading old messages, for instance. However, there is one exception - if the users use the native email application of the device, then we can measure the number of emails sent as well. If the users use a third party email application (e.g., Gmail) then we cannot say anything about the actual communication. In the remainder of the paper actions refer to application launches, voice calls made and messages sent.

We have three handset-based datasets collected between 2008 and 2010 that are used for longitudinal analysis. The data were collected in Finland with handset-based measurements conducted in the OtaSizzle project of Aalto University by installing data collection software on the participants' smartphones. Two different data collection software were used, SP360 (used, e.g., in [15]) in 2008 and MobiTrack (used, e.g., in [16]) in 2009 and 2010. Although the software were different, we were able to collect the same data types from all users. The data types collected and used in this paper include application foreground usage and voice call, SMS, MMS and native email communication (in terms of calls made/received and messages sent/received). The datasets are summarized in TABLE 1 and the users had to have at least two weeks (14 days) of active data production (i.e., usage days) in order to be included in the analysis.

All the users that participated in the handset-based measurements had a Symbian smartphone by Nokia and were students or staff of the Aalto University or its predecessor
Helsinki University of Technology. The participants were not same in all measurements, although some overlap between the participants might exist. The demographics of the users in each dataset are summarized in TABLE 2.

TABLE 1. OVERVIEW OF THE DATASETS

\begin{tabular}{llllll}
\hline & Software & Dates & Duration & \# of users & Median usage days \\
\hline Dataset 1 & SP360 & $10-12 / 2008$ & 43 days & 51 & 42 \\
Dataset 2 & MobiTrack & $10-12 / 2009$ & 71 days & 65 & 61 \\
Dataset 3 & MobiTrack & $9-11 / 2010$ & 63 days & 67 & 54 \\
\hline
\end{tabular}

TABLE 2. DEMOGRAPHICS OF THE PARTICIPANTS

\begin{tabular}{|c|c|c|c|c|c|}
\hline & Gender & Median birth year & Nationality & Occupation & Unidentified \\
\hline Dataset 1 & M (100\%) & 1983 (age 25) & Finnish (100\%) & $\begin{array}{l}\text { Stu }(98 \%) \\
\text { Sta }(2 \%)\end{array}$ & $53 \%$ \\
\hline Dataset 2 & $\begin{array}{l}M(88 \%) \\
F(12 \%)\end{array}$ & 1987 (age 22) & $\begin{array}{l}\text { Finnish }(95 \%) \\
\text { Other }(5 \%)\end{array}$ & $\begin{array}{l}\text { Stu }(75 \%) \\
\text { Sta }(25 \%)\end{array}$ & $8 \%$ \\
\hline Dataset 3 & $\begin{array}{l}M(85 \%) \\
F(15 \%)\end{array}$ & 1988 (age 22) & $\begin{array}{l}\text { Finnish }(93 \%) \\
\text { Other }(7 \%)\end{array}$ & $\begin{array}{l}\text { Stu }(79 \%) \\
\text { Sta }(21 \%)\end{array}$ & $15 \%$ \\
\hline
\end{tabular}

The participants in OtaSizzle handset-based measurements have been identified as early adopters or innovators [13] in terms of mobile devices and services [17]. Personal innovativeness in information technology has been identified as having a direct positive impact on perceived usefulness and perceived ease of using wireless Internet services via mobile technology. These perceived variables in turn have a direct positive impact on the intention to adopt these services [18]. Thus the sample is ideal for studying MI communication services as possible substitution should first be observed with these types of users. Porter [8] has also suggested that when considering substitution, companies should target early switchers as they are more likely to adopt a substitute. Von Hippel [19] has coined the term lead users who are users whose needs will reflect the situation in a marketplace months or years in the future. Furthermore, these enthusiasts or visionaries advance the state of the art of the industry, because they want to be the first in their market and are willing to take high risks [20]. If the demand of the visionaries precedes changes in the whole market, the related companies can leverage this timing advantage.

\section{RESULTS}

For analysis purposes the MI communication applications were divided to three categories - email, IM/VoIP and social media. IM and VoIP were merged to one category, because of the large amount of services that included both features (e.g., Skype and Fring). The third category includes all the social media services through which interpersonal communication is possible (e.g., Facebook and Twitter). The framework developed in [21] was used to classify all the applications in the data, with an exception of combining the messaging and calling classes to a single class called communication. The statistics were calculated with two metrics - actions and active time/duration in seconds, whereas Verkasalo [4], for example, considered only session initiations. Actions refer to application launches and calls made/messages sent, whereas active time refers to the time spent with an application active on the foreground of the device in seconds. In the case of phone calls, the duration of the call in seconds was used as an indicator of active time. We focus on the choices made by the users and thus limit the analysis to outgoing communication, whereas Gerpott [3], for example, considered both incoming and outgoing communication. 


\section{A. Descriptive statistics}

Although the datasets are not compared to each other directly and substitution will be analyzed in each dataset separately, we can compare the datasets on a general level by presenting some descriptive statistics of the datasets. The application launches in different categories are presented in Fig. 2 (cellular voice calls are not included, because they do not have a Symbian UID and are thus not considered as applications in the analysis). Fig. 2 indicates that on an aggregate level the datasets are very similar - communication, business/productivity and browsers classes dominating in all the datasets.

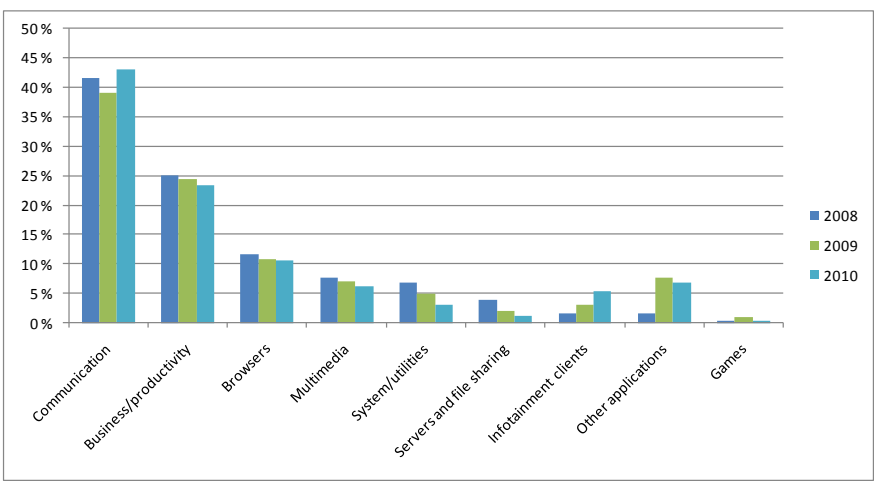

Figure 2. Launches in application classes

If we look at the communication application launches and their respective categories, we can see a bit more variation (Fig. 3). SMS dominates, but the shares of the MI applications vary among the datasets. The share of social media services is almost insignificant, because most of the usage happens through Nokia Web Runtime widgets which cannot be separated on application level with Symbian devices. However, as the widgets are implemented on top of the browser, the usage of widgets can be analyzed through HTTP (Hypertext Transfer Protocol) -traffic. For example, in the 2010 dataset $28 \%$ of total browsing was made with Facebook widgets. Further HTTP-traffic analysis will be left out for future research, however.

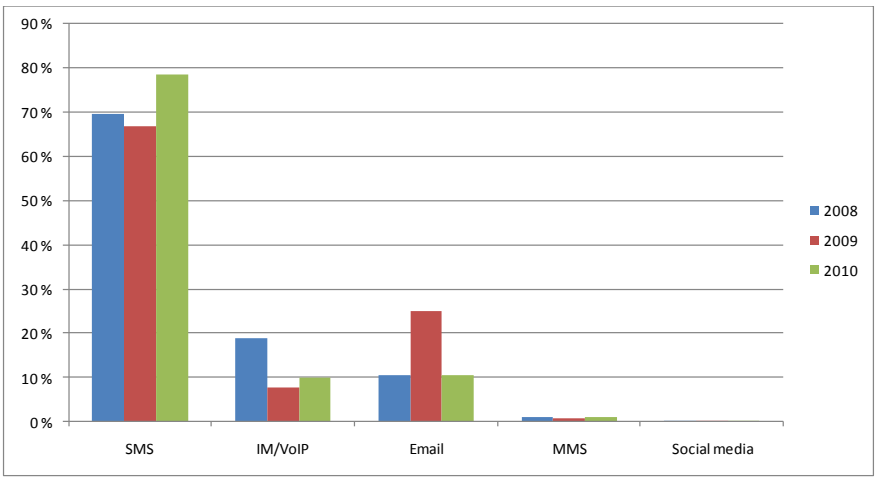

Figure 3. Launches in communication application categories

In terms of users we can look at the usage ratios of communication services (Fig. 4). Naturally both voice calls and SMSs have $100 \%$ usage ratio (with a few exceptions). $69 \%$ to $75 \%$ of the users have used MI communication services and email is the most popular MI service with a usage ratio of $61 \%$ to $71 \%$. These figures are significantly higher than the ones observed by Verkasalo [5] in 2005 and 2006 and thus studying substitution seems to be more feasible with these datasets.

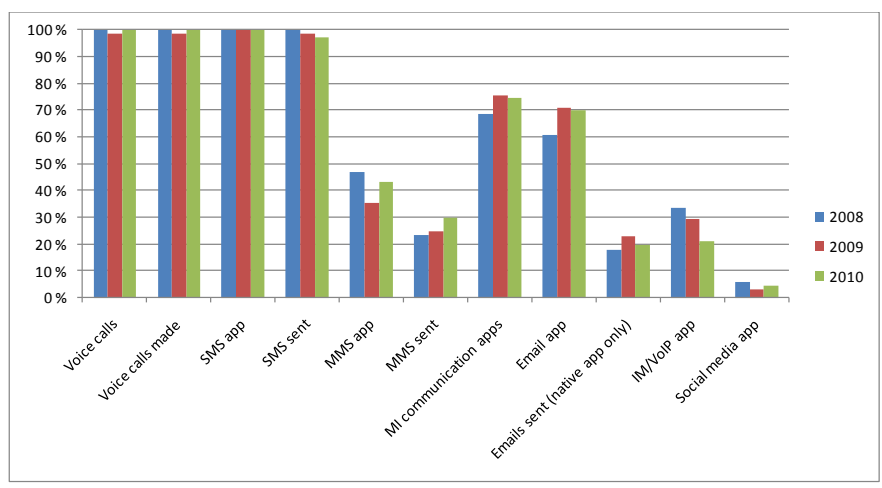

Figure 4. Communication service usage ratios

If we compare these statistics to the national level statistics of Ficora [22] the results are somewhat expected. However, it must be noted that all mobile phone users, whether they had a basic mobile phone, feature phone or a smartphone, were included in the Ficora study. Also what is considered as active usage in the Ficora study is somewhat ambiguous. In Finland $85 \%$ of mobile phone users use SMS actively, while MMS is represented by $31 \%$, personal email by $25 \%$, Internet communications (Messenger, Facebook etc.) by $20 \%$ and corporate email by $18 \%$. These figures are calculated for all age groups. If we separate these statistics by age and look at the corresponding age groups with our study (15-24 and 25-34 in Ficora's survey), it can be noted that the active usage of SMS increases to $94-95 \%$, MMS varies between $26-45 \%$, personal email between $31-42 \%$, Internet communications between 38 $39 \%$ and corporate email between $12-32 \%$. These results indicate that active use of Internet communications and personal email are typical for young adults. Our figures for email and MI communication applications in general are somewhat higher, which further strengthens the early adopter nature of our sample.

\section{B. Correlation analysis}

TABLE 3 below presents the descriptive statistics from the correlation analysis. Usage intensity is considered, meaning that actions are reported on a per day per user basis (i.e., in 2008 MI communication applications were launched 3,17 times per user per day on average). These statistics give some indication of the usage intensity of different smartphone communication services.

TABLE 3. DESCRIPTIVE STATISTICS OF THE CORRELATION ANALYSIS

\begin{tabular}{|c|c|c|c|c|c|c|c|c|c|}
\hline & \multicolumn{3}{|c|}{2008} & \multicolumn{3}{|c|}{2009} & \multicolumn{3}{|c|}{2010} \\
\hline Service & Mean & Med. & SD & Mean & Med. & SD & Mean & Med. & SD \\
\hline MI communication app launches & 3,17 & 0,81 & 6,85 & 2,62 & 0,72 & 5,89 & 2,08 & 0,44 & 3,90 \\
\hline MI communication app usage time & 55,13 & 54,05 & 31,00 & 165,99 & 48,22 & 313,85 & 112,04 & 37,62 & 292,56 \\
\hline Email app launches & 1,53 & 0,52 & 2,47 & 2,18 & 0,57 & 5,73 & 1,14 & 0,42 & 1,53 \\
\hline $\begin{array}{l}\text { Email app usage time } \\
\text { Tatitional cllts }\end{array}$ & 47,61 & 46,00 & 30,66 & 154,28 & 44,89 & 321,41 & 75,55 & 28,93 & $\begin{array}{r}255,38 \\
3,00\end{array}$ \\
\hline $\begin{array}{l}\text { Traditional callis and messages out } \\
\text { Voice calls out }\end{array}$ & $\begin{array}{l}4,56 \\
3,16\end{array}$ & $\begin{array}{l}4,24 \\
2,26\end{array}$ & $\begin{array}{l}2,68 \\
2,19\end{array}$ & $\begin{array}{l}3,70 \\
1,91\end{array}$ & $\begin{array}{l}2,92 \\
1,38\end{array}$ & $\begin{array}{l}3,17 \\
2,07\end{array}$ & $\begin{array}{l}4,08 \\
2,27\end{array}$ & $\begin{array}{l}3,58 \\
1,68\end{array}$ & $\begin{array}{l}3,09 \\
1,91\end{array}$ \\
\hline $\begin{array}{l}\text { Voice call duration } \\
\text { cilc }\end{array}$ & 143,71 & 110,25 & 119,22 & 138,92 & 134,76 & 72,86 & 171,16 & 150,80 & 124,41 \\
\hline
\end{tabular}

The datasets seem to be somewhat similar, except for the gaps between the mean values and the median values of usage times in 2009 and 2010. This indicates that the usage is quite diversified across the users in these datasets, i.e., there are lots of short usage times and a few extraordinarily long usage times. This diversity in smartphone usage has been previously 
observed in [23], for example. In the 2008 dataset this phenomenon is not visible, probably because the data collection software was different. However, when comparing the usage times of all of the datasets, the median value seems to be a better indicator of centrality than the mean, although there is concern that the average user does not represent any user.

To study substitution we conducted correlation analysis on individual user level with users that had used MI communication services. By doing this we can be sure, that the users have at least had a chance to try out the MI communication services and decide whether or not to adopt them. We studied whether the usage of MI communication services (measured both with actions and time) correlate negatively with the usage of traditional communication services.

TABLE 4. OVERALL CORRELATION ANALYSIS

\begin{tabular}{l|llll|lllll|llll|r|}
\multicolumn{1}{c}{2008} & \multicolumn{1}{c}{2009} & \multicolumn{7}{c|}{2010} \\
\hline & 1. & 2. & 3. & 4. & 1. & 2. & 3. & 4. & 1. & 2. & 3. & 4. \\
1. MI communication app launches & 1 & & & & 1 & & & & 1 & & & \\
2. MI communication app usage time & 0,08 & 1 & & & $-0,08$ & 1 & & & 0,08 & 1 & & \\
3. Traditional calls and messages out & 0,01 & $-0,06$ & 1 & & 0,06 & $-0,17$ & 1 & & 0,01 & $-0,10$ & 1 & \\
4. Voice call duration & $-0,10$ & 0,21 & $-0,39$ & 1 & $-0,06$ & $-0,21$ & 0,18 & 1 & 0,50 & $-0,03$ & $-0,09$ & 1 \\
\hline
\end{tabular}

As TABLE 4 indicates, all the correlations observed that argue for substitution (marked with bold font) on an overall level were weak $(<0.3$ or $>-0.3)$, except for one. The strong positive correlation between voice call duration and MI communication application launches in the 2010 dataset argues for complementation, but since we are focusing on substitution and the other datasets have respective correlations negative, we will not study this possible complementation further. The direction of the other correlations varies among the datasets as well. Furthermore, if we consider email specifically and separate the traditional services (TABLE 5) we can observe only weak correlations between email and the traditional communication services. In this case the directions of the correlations vary as well.

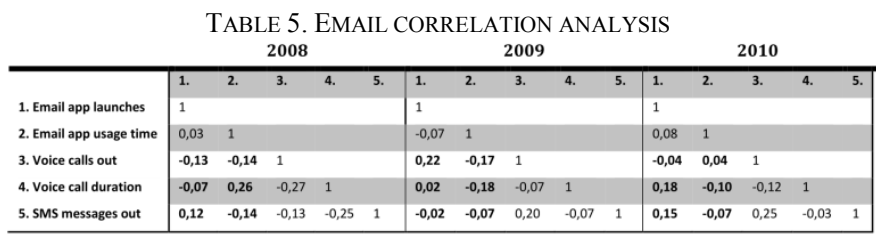

\section{DISCUSSION}

Our results support other findings in Verkasalo's [4] [5] and Gerpott's [3] [6] studies conducted with similar methods. Furthermore, not only are our observed correlations indicating substitution weak as in Gerpott's studies, but the direction of the correlations varies as well. The results also agree with other researchers who have studied the use of multiple communication media (e.g., [16] and [24]). These researchers conclude that people use different communication media for different purposes and thus, e.g., the resulting social networks and the value derived from the medium can differ quite a lot depending on the medium used. The purpose can depend, for example, on the strength of the tie or if the medium is used for maintaining or extending social networks. For example, it has been argued in [24] that in the mobile domain email is used uniformly across communities while IM/chat displays a strong community-based segregation. Furthermore, if a user uses a specific service regularly it is more likely (than what is expected based on a random adoption model) that his or her phone-based contacts use the service also.

Regarding the theoretical background part of our work, the results indicate in economic terms that the cross elasticity measures [7] between the services are near to zero. In the multiproduct interaction framework [10] the services belong into the category of independent services. However, it is unsure what will happen in the future as the services will be used by more and more people. The services might evolve to substitutes or complements in the course of time and there might also be a gradual shift in the form of reincarnation and rejuvenation as discussed previously [11]. As we are still in the beginning of the adoption S-curve [13] considering MI communication services, more usage is needed to draw conclusions on a larger scale. Also network externalities [7] will probably play a major role in the mass adoption of MI communication services and the possible future substitution on traditional communication services. The results also imply that these services might perform different, or wider/narrower, functions in the user's value activity [8] and are thus not direct substitutes. Moreover, considering the MI communication services from a technological change perspective, the results imply that we are in the variation phase [12], because the effects of the new technology are still inadequately understood. Thus there is a lot of uncertainty regarding, e.g., the market need for these services. As discussed before new technologies can influence the business of mobile operators significantly and can easily cannibalize their older services [14]. Our results imply, however, that operators should not be afraid of MI communication services cannibalizing their traditional communication services. Thus the operators should plan their marketing strategies and service offerings accordingly.

\section{A. Limitations and future research}

Regarding the external validity of the results it has to be noted, that the results are specific for the Symbian platform and we cannot generalize the results to other smartphone platforms. However, at the time of the measurements Symbian was the dominating platform in terms of market share. The results are also attributed to the early adopter group of participants, but as discussed before, their usage patterns might reflect those of the majority in the future.

The correlation analysis could be extended to multiple regression analysis as Gerpott [3] has done. He used independent variables such as MI subscription motive, MI value assessment, MI tariff type and age that were gathered using telephone interviews and the customer administration system of the operator. However, because of the limited demographic information that is available to us with these datasets, we decided not to pursue multiple regression analysis. In the future the research should be designed so that the behavioral data gathered with handset-based measurements would be complemented with questionnaires or interviews based on well-defined research questions. The study could also be extended to include more participants from multiple platforms to enable comparison between different mobile platforms, for instance. 
Bayus et al. [10] discuss a complementary portfolio of products purchased by a consumer with specific needs or desires. These products can be both substitutes and complements, because users purchase the products for different purposes and the same user can use the product differently. This portfolio aspect could be taken into account by studying how the users use different smartphone communication services together as a personal communication system [25]. This way the users could also be segmented based on the portfolios, systems or service bundles as Bouwman et al. [26] suggest. Furthermore, a study is underway, which utilizes the context detection algorithm used in [23] to study the usage of different communication services in different contexts.

As discussed previously, most of the social media service usage (e.g., Facebook) happens through Nokia Web Runtime widgets which cannot be separated on application level in Symbian smartphones. We have access to the HTTP-traffic data generated by the users, however, and can thus study the social media service usage in more detail and include the results to the correlation analysis in the future.

\section{CONCLUSIONS}

Substitution between MI and traditional communication services was studied in this paper. One could hypothesize that possible substitution should first be observed with early adopter users such as the ones participating in this research. Yet, we did not find any empirical evidence in favor of substitution. The results agree with other research conducted in this area. Thus we conclude that the smartphone communication services are used for different purposes and are not direct substitutes to each other. In the framework developed in [10] the services could thus be considered as belonging to the independent products category having only a budget relation through the finite monetary resources of the users. Furthermore, we believe that the results indicate that the saturation levels for the demand of different smartphone communication services have not been achieved yet with the current services and that there is demand for more various services. Thus the operators need to take these implications into account when planning their marketing strategy decisions and service offerings. Our results must be treated with care, however, as there are issues regarding external validity, for instance. Moreover, the new smartphone platforms such as Google Android might increase substitution in the future.

\section{ACKNOWLEDGMENTS}

The work has been supported by the OtaSizzle research project. The work was carried out in the Econ@Tel COST605 context with support from the MoMIE project and the Future Internet Graduate School (FIGS). The authors wish to thank MobiTrack Innovations Ltd. for providing the mobile audience measurement platform. The sponsoring from Nokia and Elisa to this work is also acknowledged.

\section{REFERENCES}

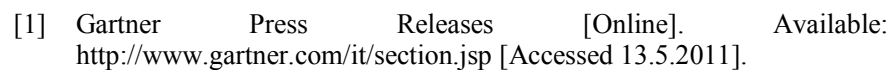

[2] Ficora, "Markkinakatsaus 1/2011: Viestintämarkkinat Suomessa 2010. Market review 1/2011: Communications Markets in Finland 2010," Finnish Communications Regulatory Authority, March 2011.
[3] T. J. Gerpott, "Impacts of mobile Internet use intensity on the demand for SMS and voice services of mobile network operators: An empirical multi-method study of German mobile Internet customers," Telecommunications Policy, vol. 34, iss. 8, 2010, pp. 430-443.

[4] H. Verkasalo, "Analysis of mobile internet usage among earlyadopters,' Info, vol. 11, no. 4, 2009, pp. 68-82.

[5] H. Verkasalo, "Handset-based measurement of smartphone service evolution in Finland," Journal of Targeting, Measurement and Analysis for Marketing, vol. 16, 2007, pp. 7-25.

[6] T. J. Gerpott, "Communication behaviors and perceptions of mobile internet adopters,' Info, vol. 12, no. 4, 2010, pp. 54-73.

[7] C. Courcoubetis, and R. Weber, "Pricing communication networks Economics, technology and modeling," New York: Wiley, 2003.

[8] M. Porter, "Competitive Advantage," Free Press, New York, 1985.

[9] P. Kotler, K. L. Keller, M. Brady, M. Goodman, and T. Hansen, "Marketing Management: First European Edition," Harlow: Pearson Prentice Hall, 2009.

[10] B. L. Bayus, N. Kim, and A. D. Shocker, "Growth models for multiproduct interactions: Current status and new directions," in V. Mahajan, E. Muller, J. Wind (Eds.) New Product Diffusion Models. Kluwer, Boston, 2000, pp. 141-163.

[11] A. D. Shocker, B. L. Bayus, and N. Kim, "Product complements and substitutes in the real world: The relevance of "other products"," Journal of Marketing, vol. 68, no. 1, 2004, pp. 28-40.

[12] P. Anderson, M. Tushman, "Technological discontinuities and dominant designs: A cyclical model of technological change," Administrative Science Quarterly, vol. 35, 1990, pp. 604-633.

[13] E. Rogers, "Diffusion of Innovations," Free Press, New York, 2003.

[14] M. McGrath, "Product Strategy for High Technology Companies," McGraw-Hill, New York, 2001.

[15] H. Verkasalo, "Handset-Based Analysis of Mobile Service Usage," Doctoral Dissertation, Helsinki University of Technology, Espoo, April 2009.

[16] J. Karikoski, and M. Nelimarkka, "Measuring social relations with multiple datasets," Accepted for publication in International Journal of Social Computing and Cyber-Physical Systems (IJSCCPS), 2011.

[17] J. Karikoski, "OtaSizzle handset-based data collection process and participant attitudes," Submitted to International Journal of Handheld Computing Research (IJHCR), 2011.

[18] J. Lu, J. E. Yao, C.-S. Yu, "Personal innovativeness, social influences and adoption of wireless Internet services via mobile technology," The Journal of Strategic Information Systems, vol 14, iss. 3, 2005, pp. 245268.

[19] E. von Hippel, "Lead users: A source of novel product concepts," Management Science, vol. 32, no. 7, 1986, pp. 791-805.

[20] G. Moore, "Crossing the Chasm: Marketing and Selling High-Tech Products to Mainstream Customers," HarperCollins, New York, 1999.

[21] T. Smura, A. Kivi, and J. Töyli, “A framework for analysing the usage of mobile services,', Info, vol. 11, no. 4, 2009, pp. 53-67.

[22] Ficora, "Viestintäpalvelujen kuluttajatutkimus 2010. Consumer survey on communications services 2010," Finnish Communications Regulatory Authority, February 2011.

[23] T. Soikkeli, J. Karikoski and H. Hämmäinen, "Diversity and end user context in smartphone usage sessions," To be presented at the 5th International Conference on Next Generation Mobile Applications, Services and Technologies (NGMAST'11), 2011.

[24] G. Szabo, and A. Barabasi, "Network effects in service usage," Arxiv preprint physics, 2006.

[25] J. Boase, "Personal Networks and the Personal Communication System," Information, Communication \& Society, vol. 11, no. 4, 2008, pp. 490-508.

[26] H. Bouwman, M. de Reuver and A. Visser, "Understanding Adoption of Mobile Service Bundles," in M. Falch and J. Markendahl (Eds.) Promoting New Telecom Infrastructures: Markets, Policies and Pricing. Edward Elgar, 2010. 